\title{
Étude de faisabilité d'un greffon biofabriqué pour traiter des récessions parodontales
}

\author{
Rawen Smirani ${ }^{1,2}$, Lucie Delattre ${ }^{1}$, Olivia Kerouredan ${ }^{1,2,3}$, Jean-Christophe Fricain ${ }^{1,2,3}$, \\ Raphael Devillard ${ }^{1,2,3}$, Adrien Naveau ${ }^{1,2,3, *}$ \\ 1 INSERM, bioingénierie tissulaire, U1026, 33000 Bordeaux, France \\ 2 CHU de Bordeaux, services d'odontologie et de santé buccale, 33000 Bordeaux, France \\ 3 Université de Bordeaux, bioingénierie tissulaire, U1026, 33000 Bordeaux, France
}

(Reçu le 11 juillet 2015, accepté le 7 octobre 2016)

Mots clés : ingénierie tissulaire / bioimpression / impression 3D / récession gingivale / hydrogel

\begin{abstract}
Résumé - Introduction : L'ingénierie tissulaire permet d'envisager de nouvelles thérapeutiques pour le traitement des récessions gingivales. Cette étude de faisabilité proposait un protocole et une chronologie pour la biofabrication de greffons parodontaux sur mesure palliant ces défauts. Technique : L'impression d'une matrice en hydrogel collagénique a été réalisée grâce à un système d'éjection piézoélectrique Microdrop ${ }^{\circledR}$. La mise au point des conditions optimales d'impression a été déterminée en fabriquant des échantillons de dimension et de morphologie compatibles avec une application clinique. L'ensemencement cellulaire de matrices a été réalisé par bioimpression assistée par laser, et la viabilité cellulaire post-impression testée. L'impression d'une matrice a été directement réalisée sur les membranes de collagène préfabriquées pour évaluer la facilité de manipulation du greffon. Les matrices obtenues ont la forme souhaitée, les cellules sont viables, et le greffon est aisément manipulable et suturable. Commentaires : L'impression de la matrice permet de choisir l'épaisseur du greffon. Il est attendu in vivo que l'organisation spatiale des fibroblastes au sein des greffons permette d'augmenter la résistance mécanique et l'esthétique des greffes parodontales. Conclusion : Cette étude de faisabilité préliminaire a permis de poser le concept. D'autres études seront nécessaires pour étudier le remodelage du greffon in vitro et in vivo.
\end{abstract}

Key words:

tissue engineering /

bioprinting / printing / three-dimensional / gingival recession / hydrogel

\begin{abstract}
Feasibility study of biofabricated graft for treating periodontal recession. Introduction: Tissue engineering is a good candidate for treating periodontal recession. This feasibility study proposed a protocol for biofabrication of customized periodontal grafts to treat this problem. Technics: Cell seeding of collagen matrices was done by laser-assisted bioprinting, and post-printing cell viability was assessed. The collagen hydrogel matrix printing was performed by a Microdrop ${ }^{\circledR}$ piezoelectric inkjet system. Optimal conditions were determined by fabricating samples whose dimensions and morphology were suitable for clinical use. Then, the hydrogel matrix printing was carried out directly on collagen membranes to evaluate the ease of the graft handling. The hydrogel matrices had the expected shape, the cells were viable, and the graft was easy to handle and suturable. Comments: The matrix printing allowed one to choose the graft thickness. Fibroblast organization was expected to improve the mechanical resistance and esthetics of periodontal grafts in vivo. Conclusion: This preliminary feasibility study established the bases of the concept. More studies are necessary to assess the graft remodeling both in vitro and in vivo.
\end{abstract}

\section{Introduction}

La récession parodontale correspond à un déplacement des tissus marginaux apicalement à la jonction amélo-cémentaire [1]. Elle touche $85 \%$ de la population adulte et favorise des hyperesthésies thermiques, des sensibilités au brossage, des caries radiculaires, des lésions cervicales d'origine non carieuse et des gênes esthétiques [2]. Le traitement repose sur l'élimination de l'étiologie, puis sur la régénération tissulaire gingivale par une intervention chirurgicale. Les principales

\footnotetext{
* Correspondance : adrien.naveau@laposte.net
}

This is an Open Access article distributed under the terms of the Creative Commons Attribution License (http://creativecommons.org/licenses/by/4.0), which permits unrestricted use, distribution, and reproduction in any medium, provided the original work is properly cited 
techniques de recouvrement radiculaire reposent sur des greffes gingivales et le déplacement de lambeaux muqueux. L'utilisation d'une membrane chirurgicale combine l'utilisation d'une membrane biodégradable et d'un lambeau déplacé coronairement [3]. Par l'unicité du site opératoire, cette technique évite les inconvénients des techniques de greffes (comorbidité du site donneur, quantité importante de tissu à prélever/déplacer pour restaurer les volumes). Toutefois, ces allogreffes guidées par membranes dermiques acellulaires (ADMA, Acellular Dermal Matrix Allograft) présentent une cicatrisation retardée et sont limitées en termes de volume de restauration [4].

Plusieurs essais cliniques ont associé des fibroblastes gingivaux autologues à des matrices afin d'améliorer le remodelage de la membrane. Les protocoles s'appuyaient sur des matrices collagéniques ensemencées de fibroblastes et une fenestration périostée, des greffes gingivales libres avec thérapie cellulaire, des ADMA ensemencées de fibroblastes associées à des greffes de tissu conjonctif (GTC) enfoui sur des récessions, ainsi que des membranes de collagène ensemencées de fibroblastes sous un lambeau déplacé coronairement [5-9]. Ces études comparant les produits d'ingénierie tissulaire (IT) avec les techniques classiques ont montré des résultats satisfaisants en termes d'inflammation postopératoire, de régénération, de couleur et de texture. Cependant, si l'adjonction de cellules améliore la vitesse de remodelage, le volume du greffon après cicatrisation reste une problématique clinique.

L'objectif de notre étude était de proposer un protocole et une chronologie de biofabrication pour des greffons potentiellement utilisables en parodontologie en combinant la technique de jet d'encre d'hydrogel pour la création de matrice avec la technique de bioimpression assistée par laser (LAB, Laser Assisted Bioprinting) pour l'ensemencement cellulaire (Fig. 1). Dans notre modèle, la matrice volumique en hydrogel collagénique reposait sur une membrane préfabriquée de collagène qui conférait au greffon sa résistance. Les cellules étaient ensemencées de manière organisée sur la matrice.

\section{Technique}

\section{Détermination des conditions optimales d'utilisation de l'imprimante à jet d'encre Microdrop ${ }^{\circledR}$}

Le mode de distribution des gouttes et le déclenchement de l'imprimante à jet d'encre piézoélectrique (Microdrop ${ }^{\circledR}$ Technologies ${ }^{\circledR} \mathrm{GmbH}$, Norderstedt, Germany) étaient contrôlés par la pression, la course du poussoir hydraulique (CPH), le temps nécessaire à l'ouverture de la valve (TNOV), le temps valve ouverte (TVO), le temps de fermeture de la valve et d'éjection de la buse (TFV), la pause entre les impulsions (PEI) et le nombre de jets par déclenchement (JD). Une goutte était faite à la demande $(G A D)$ pour $P=1$. Pour déterminer les conditions

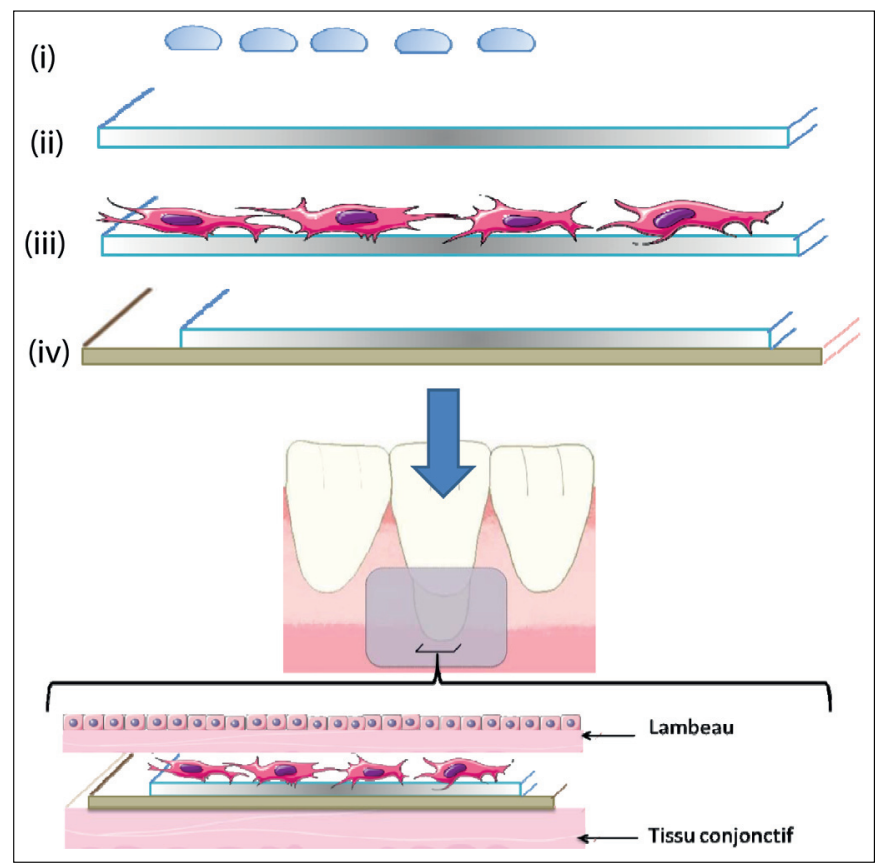

Fig. 1. Objectifs de l'étude et vue en coupe des différentes couches du greffon. (i) Essai du Microdrop ${ }^{\circledR}$ avec des gouttes à la demande. (ii) Impression d'une matrice en hydrogel collagénique. (iii) Impression de cellules par LAB sur la matrice. (iv) Impression d'une matrice sur une membrane de collagène préfabriquée.

fig. 1. Aims of the study and section view of the graft layers. (i) drop on demand printing with the Microdrop ${ }^{\circledR}$, (ii) collagen hydrogel matrix printing, (iii) cell printing on the matrix using the $L A B$, (iv) matrix printing on a collagen membrane.

optimales d'utilisation du Microdrop ${ }^{\circledR}$, tous les essais étaient réalisés par GAD avec de l'alginate sur un support plastique. L'ordinateur contrôlait le déclenchement de l'éjection de l'encre et les mouvements de la platine portant la lame réceptrice au-dessus de la buse. Le motif imprimé était des carrés en alginate $(1 \times 1 \mathrm{~cm})$, dont la réalisation a été uniforme, et conformes au schéma du set up. L'alginate à $2 \%$ était filtré et fabriqué 12 heures avant les expériences. Les valeurs nominales d'utilisation étaient : $\mathrm{CPH}=40 \%$, pression $=200 \mathrm{kPa}$, $\mathrm{TNOV}=0,5 \mathrm{~ms}, \mathrm{TVO}=0,3 \mathrm{~ms}$ et TFV $=0,5 \mathrm{~ms}$. Une seule de ces valeurs variait par expérience. Le diamètre des gouttes, mesuré au microscope optique, augmentait avec le $\mathrm{CPH}$, la pression et le TVO (pour TVO $\geq 0,3 \mathrm{~ms}$ ). Cette augmentation était proportionnelle pour le TNOV et le TFV. Les gouttes étaient significativement plus grandes sur le verre que sur le plastique.

\section{Impression d'une matrice en hydrogel collagénique sur mesure respectant la morphologie d'une récession (Fig. 2)}

Une matrice a été dessinée avec le logiciel Paint (Microsoft Corporation ${ }^{\circledR}$, Redmond WA, USA) pour correspondre à une 


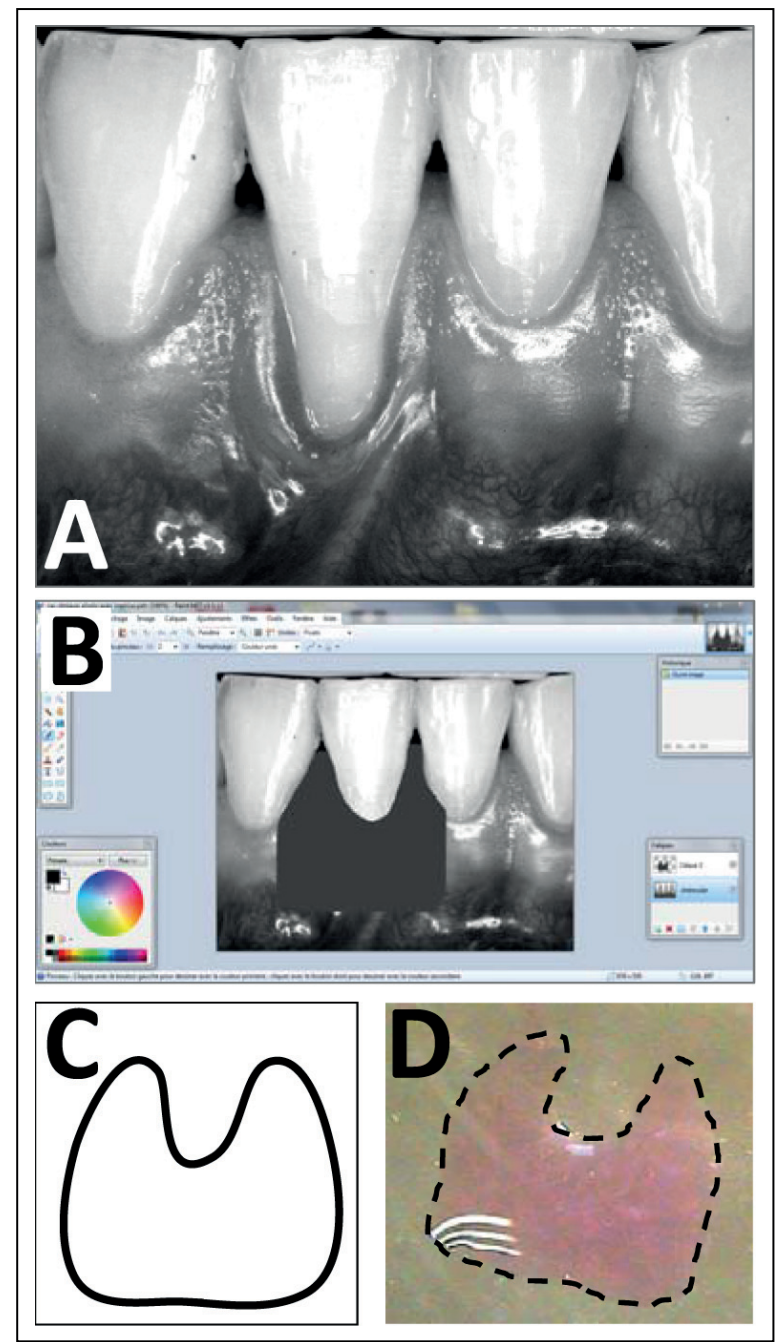

Fig. 2. Matrice en hydrogel créée au Microdrop ${ }^{\circledR}$ à partir d'un cas clinique de récession de classe II de Miller. A) Photographie clinique de la récession. B) Dessin du greffon sous Paint ${ }^{\circledR}$. C) Projet de greffon. D) Greffon imprimé.

fig. 2. Printing of the hydrogel matrix with the Microdrop ${ }^{\circledR}$ to fit a Miller class II recession. A) Clinical photograph of the recession, B) Paint ${ }^{\circledR}$ drawing of the graft, C) Graft project, D) Printed graft.

récession de classe II de Miller. En se basant sur la taille clinique de l'incisive ( $8 \mathrm{~mm}$ pour 287 pixels sur la photographie), la taille de la matrice a été déterminée $(14,8 \times 18,9 \mathrm{~mm})$. Le collagène de type I (BD Biosciences ${ }^{\circledR}$, Franklin Lakes NJ, USA) était dilué à $2 \mathrm{mg} / \mathrm{mL}$ avec du milieu Delbecco's Modified Eagle Medium (DMEM, GIBCO ${ }^{\circledR}$, InVitrogen ${ }^{\circledR}$, Carlsbad CA, USA). L'impression de la matrice en hydrogel collagénique a été satisfaisante car homogène, conforme à la morphologie choisie, et réalisée en 3 minutes. Après impression des matrices, les lames de collagène étaient placées dans l'incubateur à $37^{\circ} \mathrm{C}$ et $5 \%$ de $\mathrm{CO} 2$ pendant une heure et en présence de milieu.

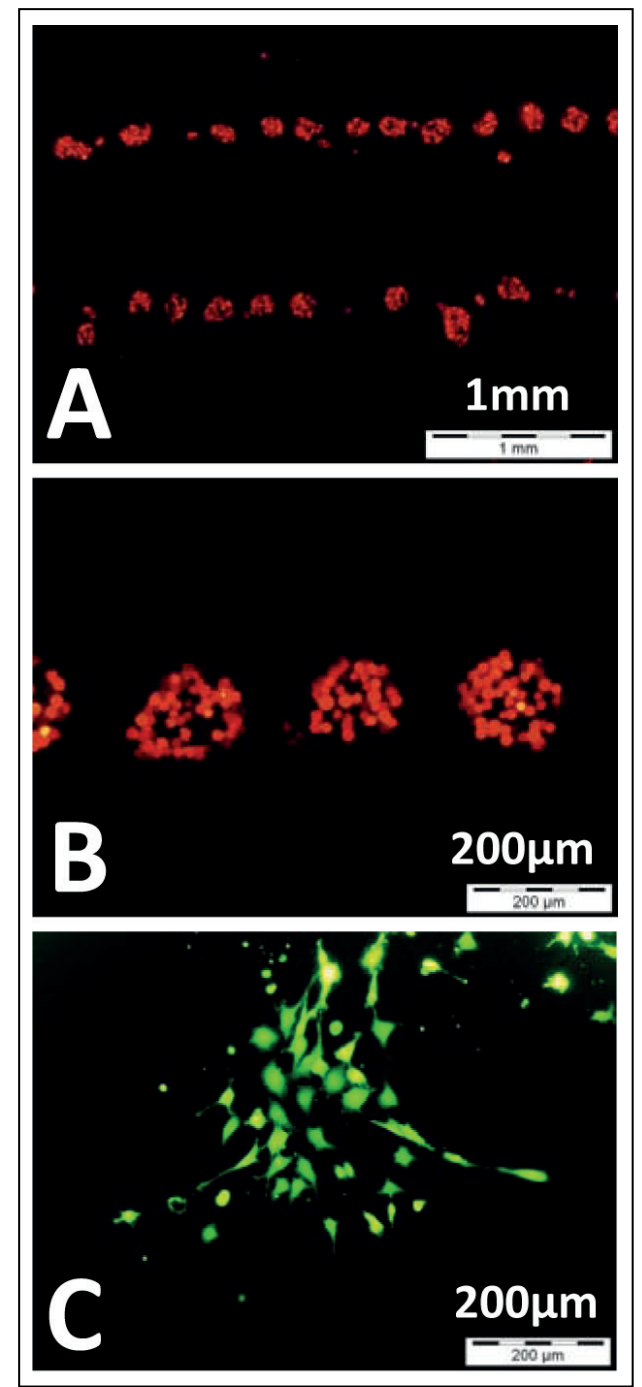

Fig. 3. Cellules D1 tdTomato à t0 post-impression au LAB en microscopie à fluorescence. A) Grossissement x 2,5. B) x 10. C) Test de viabilité à la calcéine $72 \mathrm{~h}$ post-impression (grossissement $\times 10$ ).

Fig. 3. Microscope views of $D 1$ tdTomato cells after $L A B$ printing at to A) $x 2.5, B) \times 10, C)$ calcein viability test $72 \mathrm{~h}$ post-printing $(x 10)$.

\section{Ensemencement cellulaire des matrices par LAB et survie cellulaire (Fig. 3)}

L'impression d'éléments biologiques assistée par laser a permis d'organiser des éléments cellulaires sur des matrices réceptrices. Le faisceau du laser générait ponctuellement une bulle de vapeur au sein de la bioencre et provoquait la propulsion d'une goutte sur le substrat receveur. La station de bioimpression LASIT (Laser pour l'ASsemblage en Ingénierie Tissulaire, NovaLase ${ }^{\circledR}$, Canéjan, France) avait pour source un laser $\mathrm{Nd}: Y A G$ (infrarouge $\lambda=1064 \mathrm{~nm} /$ fréquence $=1 \mathrm{kHz} /$ puissance moyenne $=19 \mathrm{mw} /$ focale $=58 \mathrm{~mm}$ ). Le motif choisi était un rectangle de $250 \times 1000 \mu \mathrm{m}$. La source était centrée 
sur le motif de la lame receveuse avec une caméra CCD à haute résolution intégrée dans le laser. Les paramètres du laser choisis étaient une puissance de $29 \mathrm{~mW}$, une vitesse de $250 \mathrm{~mm} / \mathrm{s}$ et un entrefer de $1000 \mu \mathrm{m}$.

Les cellules étaient des D1 tdTomato (D1 UVA - 0RL, American Type Culture Collection, Manassas VA, USA) (Fig. 3). Ces cellules mésenchymateuses de moelle de souris produisaient une protéine fluorescente, la tdTomato. Le milieu était du DMEM avec $10 \%$ de sérum de veau fœtal et $1 \%$ d'antibiotiques (pénicilline, streptomycine, GIBCO/InVitrogen ${ }^{\circledR}$ ) mis en atmosphère contrôlée $\left(100 \%\right.$ d'humidité, $\left.37^{\circ} \mathrm{C}, 5 \% \mathrm{CO}_{2}\right)$. Pour creer la bioencre, les cellules étaient détachées de la boîte de culture avec une solution de 0,125\% de trypsine et d'EDTA à $0,065 \%$. La concentration cellulaire obtenue était de $10^{8}$ cellules $/ \mathrm{mL}$. La lame d'or donneuse était recouverte d'une goutte de $33 \mu \mathrm{L}$ d'encre cellulaire étalée manuellement avec un embout de pipette. Les essais de bioimpression cellulaire étaient réalisés sur les matrices en hydrogel collagénique précédemment obtenues par imprimante à jet d'encre. Après l'impression, les lames receveuses étaient placées dans une boîte de 6 puits et $400 \mu \mathrm{L}$ de milieu était déposés autour des lames sans recouvrir les motifs imprimés. Après 30 minutes dans l'incubateur, $2,5 \mathrm{~mL}$ de milieu était rajouté pour recouvrir les lames. Les lames (témoins et LAB) étaient observées en microscopie photonique et en fluorescence aux temps 0,24 , 48 et 72 heures. Les lames issues du LAB étaient colorées avec un test de viabilité à la calcéine (Life Technologies ${ }^{\circledR}$, Carlsbad (A, USA) à 72 h. Les cellules étaient fluorescentes et commençaient leur étalement dès 24 heures. Les témoins ont permis de démontrer la survie cellulaire des D1 tdTomato sur les matrices de collagène issues du Microdrop ${ }^{\circledR}$ aux temps 0,24 , 48 et 72 heures.

\section{Tests de maniabilité et de suturabilité sur mâchoire de porc (Fig. 4)}

Les matrices issues du Microdrop ${ }^{\circledR}$ ont été imprimées sur une membrane collagénique préfabriquée Cova ${ }^{\circledR}$ (société Biom'up SA, Lyon, France), constituée de collagène de type 1. Avant l'utilisation, les membranes étaient réhydratées par du milieu DMEM et étalées sur une lame de verre. La matrice a été dessinée sur ordinateur à partir d'une photographie de récession gingivale sur mâchoire de porc. Il a été possible de manier et de suturer le biomatériau «membrane collagénique matrice de collagène » sous un lambeau d'épaisseur partiel avec du fils de suture résorbable (Vicryl ${ }^{\circledR}$ Plus 5-0, Ethicon Inc., Somerville NJ, USA).

\section{Commentaires}

Notre étude proposait un protocole de biofabrication pour des greffons sur mesure potentiellement utilisables en parodontologie. La technique combinait la technique de jet d'encre

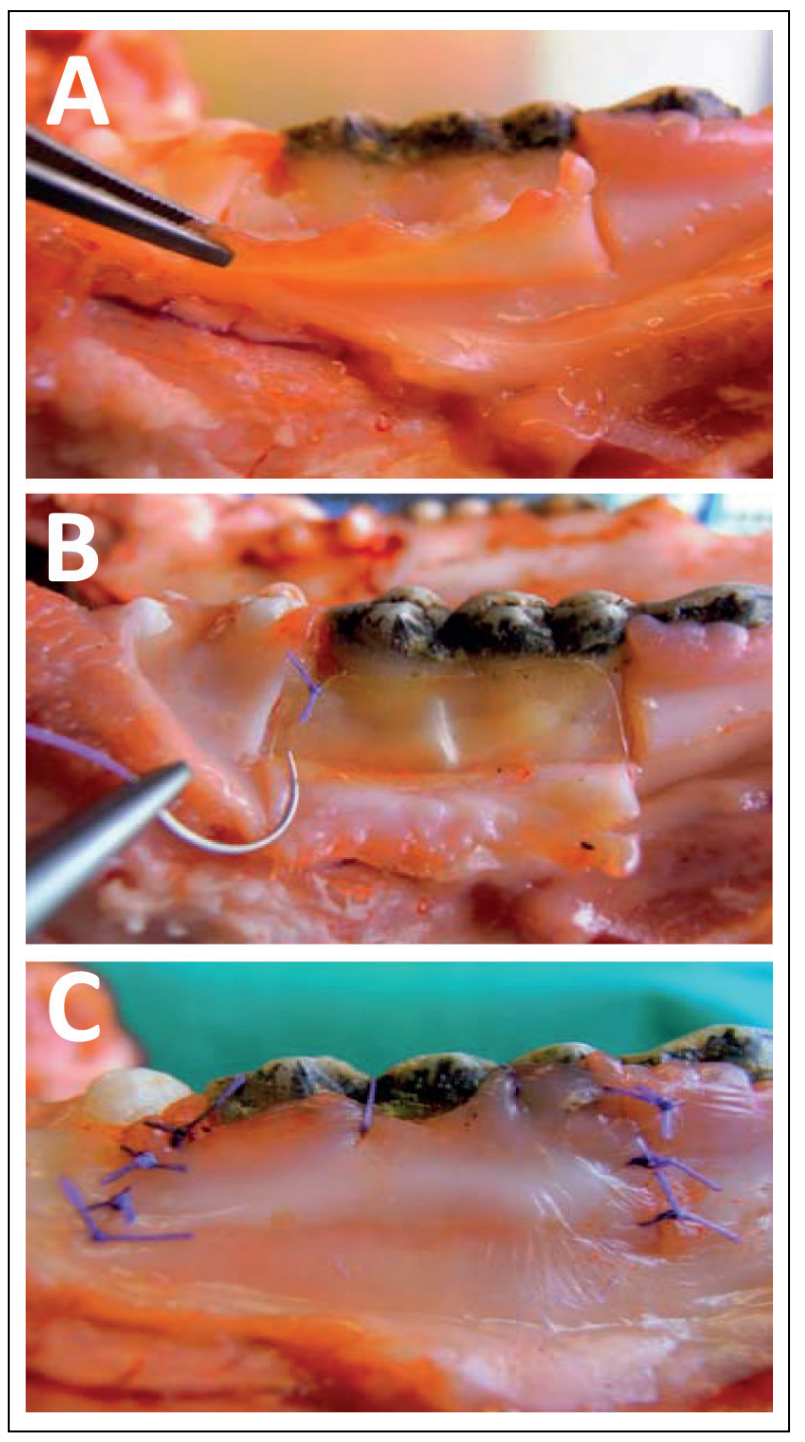

Fig. 4. Test de maniabilité et de suturabilité du greffon biofabriqué. A) Lambeau d'épaisseur partielle. B) Suture du greffon biofabriqué sur la récession. C) Suture du lambeau sur la membrane.

Fig. 4. Handling and suturing the biofabricated graft. A) Split-thickness flap. B) Suture of the biofabricated graft on the recession. C) Flap suture on the membrane.

d'hydrogel pour la création de matrice avec la technique de LAB pour l'ensemencement cellulaire. Les résultats attestaient la possibilité d'imprimer des matrices en collagène selon le volume choisi avec une imprimante piézoélectrique sur des membranes collagéniques préfabriquées. Le produit de biofabrication était manipulable pour une éventuelle utilisation clinique. D'autres études seront nécessaires pour étudier l'impact de la bioimpression sur la maintenance du volume du greffon gingival.

La Microdrop ${ }^{\circledR}$ est une imprimante à jet d'encre capable d'imprimer des cellules. Cependant, pour cette expérience, les 
contraintes en cisaillement exercées lors de l'éjection étaient trop importantes et pouvaient affecter la viabilité cellulaire. De plus, seules des solutions autour de $10^{5}-10^{6}$ cellules $/ \mathrm{mL}$ pouvaient être utilisées au jet d'encre, et cette forte concentration augmentait les risques d'obturation de l'orifice de l'aiguille [11]. Pour pallier ce problème, les matrices étaient imprimées sans cellules et ensemencées secondairement avec le $L A B$. Cette technique permet d'imprimer des solutions de 5$10 \times 10^{7}$ cellules/mL [12]. Le laser a d'autres avantages comme l'augmentation de la densité cellulaire (plusieurs passages), la combinaison de différents types cellulaires et l'organisation spatiale.

Les cellules D1 tdTomato issues du LAB ont été imprimées sur des matrices en hydrogel du Microdrop ${ }^{\circledR}$. Elles émettaient une fluorescence rouge lorsque qu'elles étaient vivantes. Ces cellules étaient colorées 72 heures après l'impression avec un test de viabilité cellulaire à la calcéine. La longueur d'onde de la tdTomato étant proche de l'homodimère-1 d'éthidium marquant la mortalité cellulaire (dead), il n'apparaissait pas judicieux d'exploiter cette donnée de mortalité. Il aurait fallu faire un test live/dead avec des cellules D1 non marquées pour définir la mortalité cellulaire post-impression à 24 et 72 heures. L'approche traditionnelle en IT parodontale ne permet pas de contrôler l'ensemencement cellulaire au sein du matériau. L'avantage du LAB était l'obtention d'une organisation cellulaire dans le substitut et la création de niches cellulaires (concentration cellulaire importante en un point précis).

Enfin, la mâchoire de porc était choisie pour des raisons de ressemblance tissulaire avec l'humain. La réhydratation de la membrane a posé un problème lors de l'impression. Lors de l'éjection sur la membrane de collagène, les gouttes s'étalaient par « dilution ». Pour pallier ce défaut, deux solutions ont été mises en place. Premièrement, la membrane, une fois hydratée et posée sur la lame de verre, était légèrement séchée. Deuxièmement, comme les gouttes étaient plus grosses sur la membrane, l'espace entre les gouttes était augmenté. Une perte de précision de la forme s'opérait lors de la découpe de la membrane autour de l'impression. Nous n'avons pas réalisé de test des produits « membrane-matrice-cellules », car l'opacité de la membrane empêchait toute observation au microscope.

\section{Conclusion}

Cette étude a montré : (i) les conditions optimales d'utilisation du Microdrop ${ }^{\circledR}$ avec des essais en D0D, (ii) qu'il était possible d'imprimer des matrices en hydrogel sur mesure respectant la morphologie souhaitée, (iii) que les cellules pouvaient tout à fait être imprimées au $L A B$ en restant viables sur ces matrices en hydrogel et (iv) qu'il était possible d'imprimer des matrices sur une membrane collagénique préfabriquée, de les manier et de les suturer. D'autres études seront nécessaires pour compléter cette étude préliminaire, comme de tester et valider in vitro la survie cellulaire dans le matériau
« membrane-hydrogel-cellules » et de confirmer cette approche in vivo sur un modèle animal.

Conflits d'intérêt : aucun

\section{Remerciements}

Les auteurs remercient l'Institut français pour la Recherche odontologue (IFRO) pour le soutien financier apporté à ce projet et à ALPhANOV pour le soutien technologique autour de la bioimpression assistée par laser.

\section{Références}

1. American Academy of Periodontology. Glossary of periodontal terms. Chicago, IL: 2001.

2. Sarfati A, Bourgeois D, Katsahian S, Mora F, Bouchard P. Risk assessment for buccal gingival recession defects in an adult population. J Periodontol 2010;81:1419-1425.

3. Wang HL, Modarressi M, Fu JH. Utilizing collagen membranes for guided tissue regeneration-based root coverage. Periodontol 2000 2012;59:140-157.

4. Novaes AB, Marchesan JT, Macedo G0, Palioto DB. Effect of in vitro gingival fibroblast seeding on the in vivo incorporation of acellular dermal matrix allografts in dogs. J Periodontol 2007;78:296-303.

5. Jhaveri HM, Chavan MS, Tomar GB, Deshmukh VL, Wani MR, Miller $P D$. Acellular dermal matrix seeded with autologous gingival fibroblasts for the treatment of gingival recession: a proof-ofconcept study. J Periodontol 2010;81:616-625.

6. Mohammadi M, Shokrgozar MA, Mofid R. Culture of human gingival fibroblasts on a biodegradable scaffold and evaluation of its effect on attached gingiva: a randomized, controlled pilot study. J Periodontol 2007;78:1897-1903.

7. McGuire MK, Scheyer ET, Nunn ME, Lavin PT. A pilot study to evaluate a tissue-engineered bilayered cell therapy as an alternative to tissue from the palate. J Periodontol 2008;79:1847-1856.

8. McGuire MK, Scheyer ET, Nevins ML, Neiva R, Cochran DL, Mellonig JT, et al. Living cellular construct for increasing the width of keratinized gingiva: results from a randomized, within-patient, controlled trial. J Periodontol 2011;82:1414-1423.

9. Köseoğlu S, Duran İ, Sağlam M, Bozkurt SB, Kırtıloğlu OS, Hakkı SS. Efficacy of collagen membrane seeded with autologous gingival fibroblasts in gingival recession treatment: a randomized, controlled pilot study. J Periodontol 2013;84:1416-1424.

10. Izumi K, Neiva RF, Feinberg SE. Intraoral grafting of tissueengineered human oral mucosa. Int J Oral Maxillofac Implants 2013;28:e295-303.

11. Saunders RE, Gough JE, Derby B. Delivery of human fibroblast cells by piezoelectric drop-on-demand inkjet printing. Biomaterials 2008;29:193-203.

12. Guillemot F, Souquet A, Catros S, Lopez J, Faucon M, Pippenger $B$, et al. High-throughput Laser Printing of Cells and Biomaterials for Tissue Engineering. Warsaw, Poland, 2008. 